\title{
Correction to: Potentially traumatic events have negative and positive effects on loneliness, depending on PTSD-symptom levels: evidence from a population-based prospective comparative study
}

Peter G. van der Velden ${ }^{1}$ Bas Pijnappel ${ }^{2}$ Erik van der Meulen ${ }^{1}$

Published online: 8 March 2018

๑) Springer-Verlag GmbH Germany, part of Springer Nature 2018

\section{Correction to: \\ Social Psychiatry and Psychiatric Epidemiology \\ https://doi.org/10.1007/s00127-017-1476-8}

In the original article, Tables 2 and 3 have been published with incorrect alignment. The correct tables are given below. The original article was corrected.

The original article can be found online at https://doi.org/10.1007/ s00127-017-1476-8.

Peter G. van der Velden

pg.vandervelden@tilburguniversity.edu

1 INTERVICT, Tilburg University, PO Box 90153,

5000 LE Tilburg, The Netherlands

2 Jacob-Roelandslyceum, Boxtel, The Netherlands 
Table 2 Prediction of loneliness among total study sample $(N=1798)$

\begin{tabular}{|c|c|c|c|c|c|c|c|c|c|}
\hline & \multirow[b]{3}{*}{$N^{\text {Total }}$} & \multicolumn{8}{|c|}{ Low versus medium loneliness } \\
\hline & & \multicolumn{4}{|c|}{$2013(\mathrm{~T} 3)^{1}$} & \multicolumn{4}{|c|}{$2014(\mathrm{~T} 4)^{2}$} \\
\hline & & $n$ & $\%^{\text {medium }}$ & AOR & $95 \mathrm{CI}$ & $n$ & $\%$ medium & AOR & $95 \mathrm{CI}$ \\
\hline \multicolumn{10}{|l|}{ Pre-event loneliness (2011) } \\
\hline Low (Ref.) & 700 & 642 & 22.3 & 1 & & 602 & 28.6 & 1 & \\
\hline Medium & 550 & 441 & 56.9 & 4.57 & $3.50-5.98 * * *$ & 412 & 52.7 & 2.72 & $2.09-3.55^{* * *}$ \\
\hline High & 548 & 197 & 64.0 & 5.92 & $4.16-8.43^{* * *}$ & 189 & 75.1 & 4.31 & $3.02-6.15^{* * *}$ \\
\hline \multicolumn{10}{|c|}{ Pre-event mental health problems (2011) } \\
\hline Low (Ref.) & 588 & 490 & 32.4 & 1 & & 471 & 36.3 & 1 & \\
\hline Medium & 616 & 451 & 44.6 & 1.62 & $1.21-2.16^{* *}$ & 417 & 44.1 & 1.32 & $0.99-1.75+$ \\
\hline High & 594 & 339 & 47.2 & 1.59 & $1.16-2.17 * *$ & 315 & 49.8 & 1.48 & $1.09-2.01 *$ \\
\hline \multicolumn{10}{|l|}{ PTE groups (2012) } \\
\hline No PTE (Ref.) & 1513 & 1076 & 41.6 & 1 & & 1010 & 43.2 & 1 & \\
\hline PTE very low PTSD-sl & 85 & 68 & 32.4 & 0.53 & $0.31-0.93 *$ & 62 & 32.3 & 0.56 & $0.32-0.99 *$ \\
\hline PTE low PTSD-sl & 69 & 50 & 34.0 & 0.67 & $0.35-1.27$ & 49 & 46.9 & 1.10 & $0.60-2.01$ \\
\hline PTE medium PTSD-sl & 80 & 56 & 32.1 & 0.71 & $0.39-1.32$ & 56 & 37.5 & 0.83 & $0.47-1.49$ \\
\hline \multirow[t]{4}{*}{ PTE high PTSD-sl } & 51 & 30 & 50.0 & 1.52 & $0.70-3.31$ & 26 & 46.2 & 1.16 & $0.52-2.60$ \\
\hline & & \multicolumn{8}{|c|}{ Low versus high loneliness } \\
\hline & & \multicolumn{4}{|c|}{2013 (T3) } & \multicolumn{4}{|c|}{2014 (T4) } \\
\hline & $N^{\text {Total }}$ & $n$ & $\%^{\text {high }}$ & AOR & $95 \mathrm{CI}$ & $\bar{n}$ & $\%^{\text {high }}$ & AOR & $95 \mathrm{CI}$ \\
\hline \multicolumn{10}{|l|}{ Pre-event loneliness (2011) } \\
\hline Low (Ref.) & 700 & 557 & 10.4 & 1 & & 528 & 18.6 & 1 & \\
\hline Medium & 550 & 299 & 36.5 & 4.74 & $3.28-6.83 * * *$ & 333 & 41.4 & 2.96 & $2.16-4.06^{* * *}$ \\
\hline High & 548 & 422 & 83.2 & 35.95 & $24.51-52.74 * * *$ & 425 & 84.5 & 19.63 & $13.81-27.91 * * *$ \\
\hline \multicolumn{10}{|c|}{ Pre-event mental health problems (2011) } \\
\hline Low (Ref.) & 588 & 429 & 22.8 & 1 & & 417 & 28.1 & 1 & \\
\hline Medium & 616 & 415 & 39.8 & 1.90 & $1.31-2.72 * * *$ & 432 & 46.1 & 1.90 & $1.37-2.63 * * *$ \\
\hline High & 594 & 434 & 58.8 & 2.69 & $1.88-3.85^{* * *}$ & 437 & 63.8 & 2.63 & $1.88-3.68 * * *$ \\
\hline \multicolumn{10}{|l|}{ PTE groups (2012) } \\
\hline No PTE (Ref.) & 1513 & 1065 & 41.0 & 1 & & 1077 & 46.7 & 1 & \\
\hline PTE very low PTSD-sl & 85 & 63 & 27.0 & 0.43 & $0.22-0.85^{* *}$ & 65 & 35.4 & 0.61 & $0.33-1.12$ \\
\hline PTE low PTSD-sl & 69 & 52 & 36.5 & 0.73 & $0.36-1.51$ & 46 & 43.5 & 0.86 & $0.43-1.72$ \\
\hline PTE medium PTSD-sl & 80 & 62 & 38.7 & 1.04 & $0.54-2.03$ & 59 & 40.7 & 0.87 & $0.46-1.64$ \\
\hline PTE high PTSD-sl & 51 & 36 & 58.3 & 2.15 & $0.93-4.95^{+}$ & 39 & 64.1 & 2.23 & $1.02-4.88^{*}$ \\
\hline
\end{tabular}

PTSD-sl PTSD symptom levels, Ref. reference group, AOR odds ratio adjusted for other predictors in table and age, gender, having a partner and education level at T2a, $N^{\text {total }}$ total number of respondents in specific category, $n$ number of respondents in specific category that endorsed low or medium, or low and high scores, \%medium percentage of respondents that endorsed medium level scores of the group with low or medium level scores, \%high percentage of respondents that endorsed high level scores of the group with low or high level scores

${ }^{+} p<0.10, * p<0.05, * * p<0.01, * * * p<0.001$

${ }^{1} 2013$ low-medium, low-high: -2 Log likehood $=1618.214, \chi(30)^{2}=763.166, p<0.0001$, Pseudo R-square Nagelkerke $=0.391$

${ }^{2} 2014$ low-medium, low-high: $-2 \log$ likehood $=1704.440, \chi(30)^{2}=579.852, p<0.0001$, Pseudo R-square Nagelkerke $=0.311$ 
Table 3 Prediction of loneliness among affected respondents $(N=283)$

\begin{tabular}{|c|c|c|c|c|c|c|c|c|c|}
\hline & \multirow[b]{3}{*}{$N^{\text {Total }}$} & \multicolumn{8}{|c|}{ Low versus medium loneliness } \\
\hline & & \multicolumn{4}{|c|}{$2013(\mathrm{~T} 3)^{1}$} & \multicolumn{4}{|c|}{$2014(\mathrm{~T} 4)^{2}$} \\
\hline & & $n$ & $\%^{\text {medium }}$ & AOR & $95 \% \mathrm{CI}$ & $n$ & $\%^{\text {medium }}$ & AOR & $95 \% \mathrm{CI}$ \\
\hline \multicolumn{10}{|l|}{ Pre-event loneliness (2011) } \\
\hline Low (Ref.) & 110 & 99 & 16.2 & 1 & & 96 & 27.1 & 1 & \\
\hline Medium & 86 & 67 & 47.8 & 5.69 & $2.58-12.51 * * *$ & 63 & 49.2 & 2.80 & $1.38-5.70^{* *}$ \\
\hline High & 87 & 37 & 64.9 & 11.56 & $4.53-29.48 * * *$ & 33 & 57.6 & 3.17 & $1.34-7.50 * *$ \\
\hline \multicolumn{10}{|c|}{ Pre-event mental health problems (2011) } \\
\hline Low (Ref.) & 101 & 85 & 27.1 & 1 & & 84 & 35.7 & 1 & \\
\hline Medium & 102 & 75 & 41.3 & 2.14 & $0.99-4.60+$ & 72 & 40.3 & 1.26 & $0.62-2.56$ \\
\hline High & 80 & 43 & 41.9 & 1.48 & $0.60-3.64$ & 36 & 47.2 & 1.45 & $0.61-3.45$ \\
\hline \multicolumn{10}{|l|}{ PTE groups (2012) } \\
\hline PTE very low PTSD-sl & 84 & 67 & 32.4 & 1 & & 61 & 32.8 & 1 & \\
\hline PTE low PTSD-sl & 69 & 50 & 34.0 & 1.15 & $0.47-2.81$ & 49 & 46.9 & 1.89 & $0.83-4.30$ \\
\hline PTE medium PTSD-sl & 79 & 56 & 32.1 & 1.42 & $0.59-3.41$ & 56 & 37.5 & 1.57 & $0.68-3.59$ \\
\hline \multirow[t]{4}{*}{ PTE high PTSD-sl } & 51 & 30 & 50.0 & 3.58 & $1.28-10.01 *$ & 26 & 46.2 & 2.16 & $0.79-5.91$ \\
\hline & & \multicolumn{8}{|c|}{ Low versus high loneliness } \\
\hline & & \multicolumn{4}{|c|}{2013 (T3) } & \multicolumn{4}{|c|}{2014 (T4) } \\
\hline & $N^{\text {Total }}$ & $n$ & $\%^{\text {high }}$ & AOR & $95 \% \mathrm{CI}$ & $n$ & $\%^{\text {high }}$ & AOR & $95 \% \mathrm{CI}$ \\
\hline \multicolumn{10}{|l|}{ Pre-event loneliness (2011) } \\
\hline Low (Ref.) & 110 & 94 & 11.7 & 1 & & 84 & 16.7 & 1 & \\
\hline Medium & 86 & 54 & 35.2 & 4.63 & $1.87-11.49 * * *$ & 55 & 41.8 & 3.44 & $1.48-8.01 * *$ \\
\hline High & 87 & 63 & 79.4 & 28.94 & $11.11-74.42 * * *$ & 68 & 79.4 & 16.04 & $6.60-39.01 * * *$ \\
\hline \multicolumn{10}{|c|}{ Pre-event mental health problems (2011) } \\
\hline Low (Ref.) & 101 & 78 & 20.5 & 1 & & 71 & 23.9 & 1 & \\
\hline Medium & 102 & 71 & 38.0 & 2.61 & $1.09-6.21^{*}$ & 73 & 41.1 & 2.01 & $0.91-4.81+$ \\
\hline High & 80 & 62 & 59.7 & 3.78 & $1.50-9.51 * *$ & 63 & 69.8 & 4.34 & $1.76-10.72 * * *$ \\
\hline \multicolumn{10}{|l|}{ PTE groups (2012) } \\
\hline PTE very low PTSD-sl & 84 & 62 & 27.4 & 1 & & 64 & 35.9 & 1 & \\
\hline PTE low PTSD-sl & 69 & 52 & 36.5 & 1.63 & $0.62-4.34$ & 46 & 43.5 & 1.44 & $0.57-3.61$ \\
\hline PTE medium PTSD-sl & 79 & 61 & 37.7 & 2.25 & $0.89-5.77+$ & 58 & 39.7 & 1.42 & $0.58-3.45$ \\
\hline PTE high PTSD-sl & 51 & 36 & 58.3 & 5.24 & $1.76-15.58^{* *}$ & 39 & 64.1 & 3.13 & $1.12-8.69 *$ \\
\hline
\end{tabular}

The total number of affected respondents is lower than in Table 1 (285 versus 283), because for two respondents the education was unknown and, therefore, were omitted from the analyses

$P T S D$-sl PTSD symptom levels, Ref. reference group, AOR odds ratio adjusted for other predictors in table and age, gender, having a partner and education level at T2a, $N^{\text {Total }}$ total number of respondents in specific category, $n$ number of respondents in specific category that endorsed low or medium, or low and high scores, \%medium percentage of respondents that endorsed medium level scores of the group with low or medium level scores, \%high percentage of respondents that endorsed high level scores of the group with low or high level scores

$+p<0.10, * p<0.05, * * p<0.01, * * * p<0.001$

${ }^{1} 2013$ low-medium, low-high: $-2 \log$ likehood $=428.413, \chi(26)^{2}=127.000, P<0.0001$, Pseudo R-square Nagelkerke $=.411$

${ }^{2} 2014$ low-medium, low-high: $-2 \log$ likehood $=452.678, \chi(26)^{2}=112.724, P<0.0001$, Pseudo R-square Nagelkerke $=.371$ 